\title{
ARTICLE \\ Design of UAV Level Control Method
}

\section{Dexuan Luo, Ruixuan Zhang, Siying Wang, Qun Ding*}

Zhujiang College, South China Agricultural University, Guangzhou, Guangdong, 510900, China

\begin{tabular}{l}
\hline ARTICLE INFO \\
\hline Article history \\
Received: 28 May 2019 \\
Revised: 10 June 2019 \\
Accepted: 10 October 2019 \\
Published Online: 16 October 2019 \\
\hline Keywords: \\
UAV level control \\
UAV control \\
UAV \\
Drone
\end{tabular}

\section{Introduction}

$\mathrm{I}$ $\mathrm{n}$ the era of quantitative change in science and technology, human's pursuit of quality has become more and more standardized. Therefore, high-end products have been more and more popular and valued, which has gradually become a strong market trend. UAV, a high-tech product, is well known and applied by more and more people. It integrates power and control ${ }^{[1]}$, with advantages like low

\begin{abstract}
Starting from the current problems of drones, this paper aims to find solutions that are suitable for sustainable development. This paper mainly introduces a method of adjusting the control authority of UAV, including managing the process, obtaining the operation of maneuvering performance and controlling the accuracy rate. This method enhances the fault tolerance of UAV flight control, improves the high precision of UAV flight control, and avoids the occurrence of accidents caused by improper control, such as "drone damage" or "personal injury to others".
\end{abstract}

cost, zero casualty, reusability and high mobility.

With the widespread use of drones, how should drone operators standardize drone operations? How should drones be used professionally for operations? Whether there are solutions to flight safety problems of drones has also attracted the attention of many people. And how can drone operators solve the problems that new drone operators are likely to "blow up" the planes in a safer way. This paper

*Corresponding Author:

DingQun (1981-),

Female, a native of Changde in Hunan Province, China, lecturer, master degree;

Research direction: computer science and technology;

Correspondence address: Zhujiang College, South China Agricultural University, No. 72 Guangcong North Road, Conghua District, Guangzhou, Guangdong, China;

E-mail: 381705049@qq.com.

Fund Project:

This paper is funded by the special construction project of "Innovation Strong School Project" in Guangdong Province and Zhujiang College, South China Agricultural University. The Project name is "Destructive innovative tourism products based on UAV AR Interactive Game" (Project No.: 201812623005S); This paper is also funded by the Higher Education Teaching Reform Project in 2017 (No. 706), namely The application, exploration and practice of WeChat public platform in college curriculum teaching under the environment of mobile Internet Plus. 
will look for solutions from a technical point of view.

\section{UAV Background Introduction}

As the name suggests, Drones, also knowns as remote-controlled driving vehicles, are aircrafts that are not operated by human pilots inside the flight is entirely dependent on the operator's remote proximity control on the ground ${ }^{[2]}$. The fuselage has automatic operating systems, such as program control systems, remote control and telemetry systems, to achieve all-round UAV flight control ${ }^{[3]}$.

With the combination of various functions and industries, drones have owned a relatively complete industrial structure. UAV industry chain can be divided into research, development, manufacturing, product sales and product services. The increasing total amount and enhancing quality of drones will drive a large number of users and equipment in the future gathered together to form communities like aerial flight circle, air sports circle and so on. Moreover, integration effect, aggregation effect, synergy effect of the industrial value chain will be motivated ${ }^{[4]}$. It is the drive of market demand that makes drone gradually public ${ }^{[5]}$.

UAV is now mainly used in military and civilian fields and the proportion of the use in civilian fields is constantly increasing. In the civilian field, drones can be applied in border patrols, resource exploration, disaster reconnaissance, communications relays, environmental monitoring, etc $^{[1,2]}$. In addition, drone aerial photography can enhance the texture of the picture shooting, providing the film and television industry with production of irreplaceable visual effects.

With the development of UAV industry, its related service market is gradually growing and the industrial chain will be expanded. From the point of view of market size, the UAV Service market will have great investment value in the future.

\section{Existing Problems and Preliminary Solu- tions to UAV Flight}

\subsection{Existing Problems of Drones}

In recent years, the UAV industry has entered a stage of rapid development under various favorable factors. However, there is still detention of permission requirements of operators and blurred boundary between professional operators and novice operators. Additionally, the flight boundaries of drones are still unclear and most of the rules and regulations are not perfect ${ }^{[6]}$. At the same time, the growth rate of the number of drones is not proportional to the growth rate of their professional operators. This condition leads to a lack of knowledge of UAV flight policy and flight operation for novice drone operators, which may cause accident due to careless operation.

Every year, there are incidents in the world that affect the normal operation of the airport due to improper use of drones. In recent years, there are some illegal drone flight incidents occurred in some airports in China that have serious effects. For example, at Hangzhou International Airport, a drone broke into the airport clearance reserve, interfering with the normal flight of the aircraft and affecting the flight safety of the aviation ${ }^{[7]}$. Such incidents not only pose a threat to air transport flight safety but also have an impact on social public safety ${ }^{[8]}$. The usual treatment for such incidents is the restriction of drones' flight in some areas or the use of UAV counterattack gun to shoot down the aircrafts. These initiatives can reduce illegal drone flight in a short period of time, but in the long run, it is worth pondering how much these initiatives can regulate drone flight chaos.

Nowadays, the UAV is controlled by remote controllers, and the UAV operators can decide the flight height or speed in non-restricted flight areas of their own initiatives. Such control methods with free control rights are poor in fault tolerance and are easy to cause accidents due to improper control in non-restricted flight areas, such as damage to drones or personal injury to others.

\subsection{Preliminary Solutions to UAV Flight Problems}

In view of the flight control defects of the existing UAV mentioned above, a method of UAV level control authority comes into being, which provides guarantee for the new operators in UAV operation. Its permissions include speed permissions, distance permissions, and one or more features in the control dimension permissions. It can be divided into four steps.

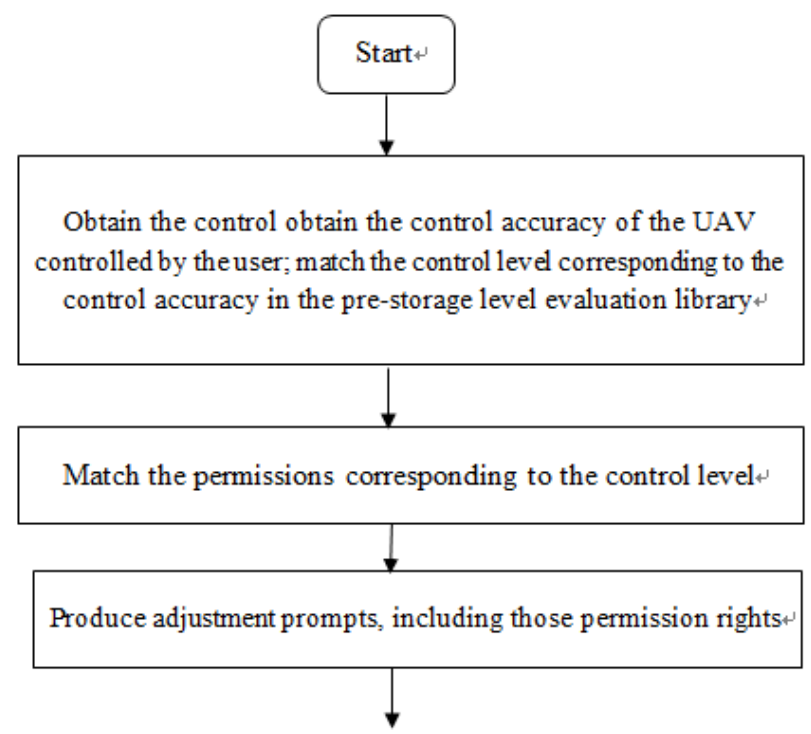

DOI: https://doi.org/10.26549/met.v3i2.1824 
Adjust the control authority of UAV to the permission, so

that the users will control the UAV according to the control authority to operate the flight correspondingly

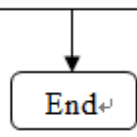

Figure 1

Step one is to obtain the control accuracy of the UAV controlled by the user, and to match the control level corresponding to the control accuracy in the pre-storage level evaluation library. Step two is to match the license permissions corresponding to the control level. And step three is output of adjustment prompts, including those of permission rights. While in step 4, The current control authority of the UAV is adjusted to the permission, so that the users will control the UAV according to the control authority to operate the flight correspondingly. After this step, the method also includes repeating step one to step four per preset time period to adjust the control permissions of the UAV.

The above is a preliminary solution to the defects of UAV operation flight. This method gradually carries on the corresponding matching dispatching for novice UAV operators, making it possible for them to use UAV in a more accurate way and avoid safety accidence caused by mis-operation.

\section{The Concrete Implementation Mode of UAV Level Control Method}

In order to interpret this paper more clearly, the following will be a logical and comprehensive description of the technical methods of this paper through UAV manipulation embodiments and drawings.

\subsection{The procedures of drone control permission method}

Firstly, the control accuracy of the user control UAV is obtained, and the control level corresponding to the control accuracy is matched in the pre-storage level evaluation library. The actuator of the adjustment method of UAV control authority can be the adjustment device of UAV Control authority, the control device of UAV, the mobile devices, terminal devices, etc. The adjustment method of the drone control authority of the service is applied to the adjustment devices mentioned above, and the control accuracy of the UAV can be obtained by the user under the adjustment device when the user first initiates the control of UAV.

Next, it will match the permissions that correspond to the control level. The control level includes four levels, namely entry, elementary, intermediate, and advanced levels. Licensed permissions include controlling the maximum flight speed of UAV flight, controlling the farthest distance of UAV flight, controlling the control dimensions of UAV flight and so on. The control dimension includes controlling the back-and-forth, up-and-down and left-andright movement of UAV. And it can also include the controlling of rotation of UAV.

The next step is producing permission adjustment prompts that include the licensed permission. Finally, the current control permissions of the UAV are adjusted to licensing permissions so that the user controls the drone to perform the corresponding flight operation according to the control rights. When the control instructions entered by the user are received, it will determine whether the control permissions of the current drone include the permissions corresponding to the control instruction. If included, it control the drone to perform the corresponding flight operation according to the control instruction. If not included, the instruction should not be followed and the No-action permission prompt will be produced. After they are confirmed as control permissions for the drone, it can determine the current unlicensed permissions based on both the permissions and the full permissions that are stored. Based on the current unlicensed permissions, the current unlicensed permissions will automatically control drone flight.

\subsection{Methods for Obtaining Accuracy Rate of UAV Control}

With regard to the acquisition of the accuracy method of UAV control, it is necessary to obtain the control accuracy of the UAV controlled by the user according to the characteristics of the UAV Control permission adjustment methods. This includes obtaining the dynamic flight pictures on the UAV, virtual dynamic images, virtual flight route images and dynamic flight images generated from the sensor data collected by various sensors on UAV. And the augmented reality screen are produced and shown based on the virtual dynamic images and virtual flight route images. The actual route of the UAV flight is shown on the augmented reality screen, and the coincidence rate between the actual route and the virtual flight route is determined as the control accuracy rate.

Firstly, get the current flight route and determine the coincidence rate between the flight route and the preset route.in order to determine the coincidence rate, it includes obtaining the flight view and flight route of the $\mathrm{UAV}$, superimposing the preset route in the flight view 
and obtaining the virtual reality view, where the flight route is displayed and the coincidence rate between the flight route and the preset route can be determined.

Then the corresponding error correction model is obtained according to the preset route, and the control accuracy rate obtained by using error treatment of coincidence rate according to the error correction model, The error correction model is used to compare the actual flight route with the preset one and obtain the coincidence rate. After that, it can determine whether the coincidence rate is lower than the preset threshold. If it is lower, it suggests that the coincidence rate of the flight route and the preset route is relatively low. Through adjusting the value of error band width in the error correction model, which means increasing the line width of the preset route, and comparing the flight route with the adjusted preset one, the corrected coincidence rate is obtained. Then, whether the difference between the corrected coincidence rate and the original coincidence rate exceeds the change threshold should be decided. If it exceeds the change threshold, it indicates that there is mutation of the modified coincidence rate, therefore the flight route is abnormal, which needs to be re-determined. If it doesn't exceed the change threshold, it indicates that the change of corrected coincidence rate is normal and the corrected coincidence rate will be used as the control accuracy rate. The greater the error bandwidth in the error correction model, the higher accuracy of obtaining users' control accuracy rate of controlling UAV. The size can either be set by the user or automatically set by the adjustment device.

\subsection{UAV Control Accuracy Calculation Process}

Before the flight, the system will determine the flight route based on the user's chosen pass points and define the route as the perfect track for drone flight. At the same time, the user input UAV flight preset width $x_{0}$, flight accuracy $p$ is the relative ratio of preset flight effect and actual flight effect the moment the plane takes off. According to the definition, the accuracy rate of UAV flight within the preset width is calculated by $100 \%$.

During the flight of the drone, the system will obtain the real-time deviation value $x$ of the UAV and the instantaneous velocity $v$ at the current direction of the perfect orbit every $\Delta t$ time. Therefore, at every $\Delta t$ moment, the real-time coincidence rate $c$ and flight distance of the drone $s$ will be calculated. $c=\frac{x_{0}}{x} \times 100 \%, s=v \times \Delta t$.

If the distance the UAV deviates from the perfect track is longer, the coincidence rate and accuracy rate of flight will be lower. So it can be suggested that the accuracy of the UAV flight over a period of time is related to the real-time orbital width of the flight. And the same coincidence rate can be maintained if the UAV flies in the same orbit in the direction of the perfect orbit. If the flight distance is different, the accuracy of the flight will be not the same. Taken together, we can conclude that the flight accuracy of the drone is related to the distance of its forward flight.

To sum up, according to the various contributory factors in the flight process, the relations among the accuracy rate every $\Delta t$ moment, the coincidence rate (that is, the real-time flight track width) and the distance of is forward flight can be concluded as:

$$
p_{i}=\frac{p_{i-1} \times S_{i-1}+c_{i, 1} \times s_{i, 1}+c_{i, 2} \times s_{i, 2}+\cdots+c_{i, m} \times s_{i, m}}{S_{i-1}+S_{i}}
$$

$p_{i}$ is the accuracy rate of the $i^{\text {th }}$ time. $c_{i, 1}, s_{i, j}$ $\left(j=1,2, \cdots, m, m=\frac{\Delta T}{\Delta t}\right)$ indicates the $i^{\text {th }}$ time coincidence rate and the calculated real-time flight distance according to the $i^{\text {th }}$ time coincidence rate. $S_{i}=S_{(i, 1)}+s_{(i, 2)}+\ldots+s_{(i, m)}$ suggests the flight distance along the perfect track during the period between the $i-1^{\text {th }}$ time and $i^{\text {th }}$ time accuracy rate.

The control accuracy of the $i^{t h}$ time flight is judged by the correlation among flight accuracy, permission and control level derived from the -1 th time.

If the $i-1^{\text {th }}$ time control level is 2 and $p_{i}<50 \%$, it will adjust error broadband, which means adding the preset orbit width $\Delta x=\frac{x-x_{0}}{2}$. If $\frac{\Delta x}{x}>0.2$, the system will give a degrading prompt. Otherwise, the existing level 2 will be maintained. However, if $p_{i}<50 \%$, level 2 will be maintained.

If the $i-1^{\text {th }}$ time control level is 3 and $p_{i}<70 \%$, it will adjust error broadband, which means adding the preset orbit width $\Delta x=\frac{7 x-10 x_{0}}{10}$. If $\frac{\Delta x}{x}>0.15$, the system will give a degrading prompt. Otherwise, the existing level 2 will be maintained. However, if $p_{i}<70 \%$, level 3 will be maintained.

If the $i-1^{\text {th }}$ time control level is 4 and $p_{i}<85 \%$, it will adjust error broadband, which means adding the preset orbit width $\Delta x=\frac{85 x-100 x_{0}}{100}$. If $\frac{\Delta x}{x}>0.15$, the system will give a degrading prompt. Otherwise, the existing level 2 will be maintained. However, if $p_{i}<50 \%$, level 4 will be maintained.

\subsection{The Relations Among Control Accuracy Rate, Control Level And Permission}

The corresponding relations among control accuracy rate, control level and permission are as follow. When the control accuracy is $\mathrm{P}<50 \%$, the maximum flying speed for permission is $3 \mathrm{~m} / \mathrm{s}$, the farthest control distance is $150 \mathrm{~m}$ and the control level is 1 when controlling the forward movement of the dimension. The detailed relations of control level 2, 3 and 4 are shown in Figure 2. 


\begin{tabular}{|c|c|c|c|c|}
\hline \multirow{2}{*}{$\begin{array}{c}\text { Control Accu- } \\
\text { racy Rate(p) }\end{array}$} & \multirow{2}{*}{$\begin{array}{c}\text { Control } \\
\text { Level }\end{array}$} & $\begin{array}{c}\text { Maximum } \\
\text { Flying Speed }\end{array}$ & $\begin{array}{c}\text { Max- } \\
\text { imum } \\
\text { Distance }\end{array}$ & Control Dimension \\
\hline $\mathrm{p}<50 \%$ & 1 & $3 \mathrm{~m} / \mathrm{s}$ & $150 \mathrm{~m}$ & back-and-forth movement \\
\hline $50 \%<\mathrm{p}<70 \%$ & 2 & $5 \mathrm{~m} / \mathrm{s}$ & $300 \mathrm{~m}$ & $\begin{array}{c}|c| \\
\text { back-and-forth movement, left- } \\
\text { and-right movement }\end{array}$ \\
\hline $70 \%<\mathrm{p}<85 \%$ & 3 & $10 \mathrm{~m} / \mathrm{s}$ & $600 \mathrm{~m}$ & $\begin{array}{c}\text { Permissions } \\
\text { back-and-forth movement, left- } \\
\text { and-right movement, up-and- } \\
\text { down height adjustment }\end{array}$ \\
\hline $85 \%<\mathrm{p}<100 \%$ & 4 & $15 \mathrm{~m} / \mathrm{s}$ & $1500 \mathrm{~m}$ & $\begin{array}{c}\text { deft-and-right movement, up- } \\
\text { and-down height adjustment, } \\
\text { diagonal movement }\end{array}$ \\
\hline
\end{tabular}

Figure 2

The user can perform the matching flight operation of the UAV according to the control rights or manually adjust the control permissions of the current UAV, while control level corresponding to the control permissions that the user can manually adjusts can only be less than or equal to the control level corresponding to the current control permissions. For example, assuming that control permissions are divided into four levels, which are entry, elementary, intermediate, and advanced level, and that the control level for the current control permissions is intermediate, the control permissions that the user can switch manually can be of entry, elementary and intermediate level.

\section{Conclusion}

To sum up, this paper puts forward a UAV level control method which can improve the safety factor of UAV operators from the analysis of the general management problem of UAV under the background of the development of UAV industry nowadays. This paper discusses in detail the process of the method, how to obtain and calculate the accuracy of the method, and then determine the flight grade of the UAV operator. Additionally, the safe use of the UAV can be guaranteed effectively by matching the UAV operators and their flight control degree accordingly based on the relations among control accuracy rate, control level and permissions.

\section{References}

[1] Li Yunqi. New direction and prospect of UAV technology application[J]. Private technology, 2018(a).

[2] Maryam Asadzadeh Kaljahi, Palaiahnakote Shivakumara, Mohd Yamani Idna idris, Mohammad Hossein Anisi, Tong Lu, Michael Blumenstein, Noorzaily Mohamed Noor. An automatic zone detection system for safe landing of UAVs[J]. Expert Systems with Applications, 2019(122).

[3] Li Luchi. UAV Transverse attitude Control Research[D]. Central South University.

[4] Tan Qing, Ma Junhua, Jhonychoon Yeong Ng. Development approach and strategy of civil-military integration of UAV industry based on " three chain "[J/OL]. Scientific and technological progress and Countermeasures: 1-8 [2019-05-06]. http://kns.cnki. $\mathrm{net} / \mathrm{kcms} /$ detail/42.1224.G3.20190424.1621.012. $\mathrm{html}$.

[5] Shiying, Li Guiqing. Analysis of development environment of civil UAV industry in China based on PEST Analysis[J]. Modern, 2017, Vol.07 (06), pp.400-405 Hans Press.

[6] Wang Sen. On the harm of UAV black flying to civil aviation flights $[\mathrm{J}]$. Economic and trade practice, 2017(a):329.

[7] Liu Yishui. When drones sail into the " no-fly zone “[J]. Chinese Entrepreneur, 2015(07):116-117.

[8] Hadmade Hector. Impact of drones on airports and recommendations for response $[\mathrm{J} / \mathrm{OL}]$. Electronic technology and Software engineering, 2019(08): 108109 [2019-04-30].

http://kns.cnki.net/kcms/detail/10.1108. TP.20190428.1405.146.html.

[9] Zhang Yu won. The drone "black fly" cannot stop at shooting down[N]. China Civil Aviation Daily, 201809-21(003). 